\title{
Hemipelvectomy for liposarcoma: an unusual case and course
}

\author{
ALEXANDER A. DEUTSCH \\ M.B., Ch.B., F.R.C.S. (Eng), F.R.C.S. (Edin) \\ AVRAHAM BACHAR \\ M.D. \\ RAPHAEL REISS \\ M.D.
}

Department of Surgery B, Beilinson Medical Center, Petah Tiqva 49100 and Tel Aviv University Sackler Medica 5 School, Israel

\begin{abstract}
Summary
A patient with liposarcoma of the upper thigh and pelvis treated by hemipelvectomy is described. Preoperative treatment with radiotherapy and chemotherapy induced regression and necrosis of the growth. The treatment of this tumour is discussed as well as the possibility that the pre-operative therapy converted an inoperable tumour into an operable one.
\end{abstract}

KEY WORDS: liposarcoma, pelvis, hemipelvectomy, radiotherapy, fluorouracil.

\section{Introduction}

Most soft tissue sarcomas are tumours of high local malignancy; liposarcomas are particularly malignant (Miller, 1977). The treatment of this tumour should be radical and aggressive even though involving very wide excisions (Krementz and Shaver, 1963). When the tumour involves the buttock, hip and upper thigh a radical operation offers the best chance of cure. The operation of hemipelvectomy in a reasonably fit patient carries a very acceptable mortality and morbidity rate. Miller (1977) reports a mortality rate of only $1.5 \%$ in a series of 126 cases. Douglass, Razack and Holyoke (1975) had no mortality in 50 patients.

We report a case of liposarcoma of the left thigh and pelvis in which the disease was thought to be incurable and inoperable. After a course of radiotherapy and chemotherapy, bleeding of the tumour mass resulted in a reassessment of the patient and a left hemipelvectomy was successfully performed. The patient is alive and well 3 years after surgery with no evidence of recurrence.

\section{Case report}

A 46-year-old male was admitted with a mass in the left inguinal region. The lump had grown rapidly over the previous 6 months, but the patient ha previously refused treatment.

There was no weight loss. The left groin showed a mass of $20 \times 20 \mathrm{~cm}$, hard, fixed and painless which extended half-way down the thigh, and disappeared proximally beneath the inguinal ligament (Fig. 1 E There was some mild oedema of the left leg, and absent peripheral pulses on this side.

A radiograph of the area showed the massot contain irregular areas of calcification, both in the periphery as well as in the centre (Fig. 2). A biops af the mass revealed a partially calcified gelatinou tumour and histology was reported as a liposarcom of the mixed type.

Investigations performed included routine bloo and urine tests, and liver, spleen and bone scans which were all normal. Computerized tomography of the mass and abdomen indicated that tumour reached the level of the anterior superior iliac spine

The tumour was at this stage thought to be inoperable, and he was therefore referred for radio 3 therapy. Treatment comprised 5000 rads in a regimeof two doses of 300 rads daily, after sensitization with. 5 -fluorouracil. There was obvious clinical regressiog of the mass. However, the biopsy site broke dow and a necrotic discharge continued over a period of months.

The patient was admitted as an emergency months later with severe exanguinating arteria haemorrhage from the necrotic tumour of the lef thigh. Cessation of the bleeding could only be achieved by ligation of the left external iliac artery int addition to local suture. At surgery it was noted that no tumour was present in the pelvis. The left leg became partially ischaemic, and the tumour are necrotic and it was decided to perform a radicas excision of the tumour by means of a left hemipelvectomy. 


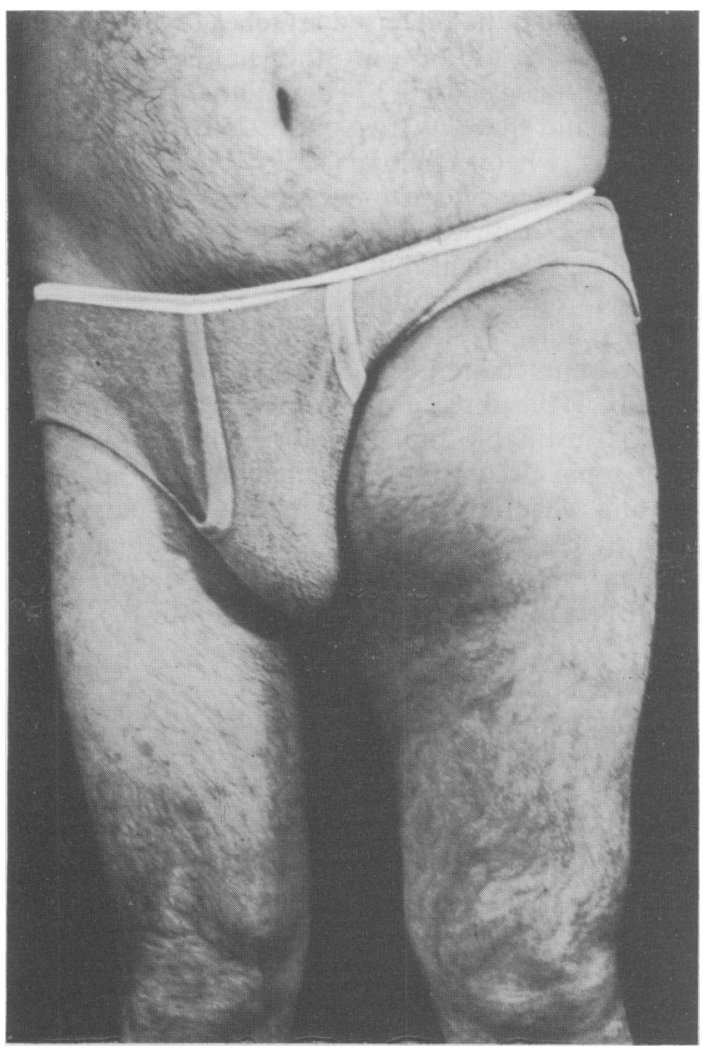

FIG. 1. Liposarcoma of the left thigh on presentation. The tumour disappeared beneath the inguinal ligament proximally.

The operation involved removal of the left lower limb in continuity with the left pelvis. The ileum was transected $6 \mathrm{~cm}$ from the left sacroiliac joint, and the pubis was divided at the symphysis.

The patient made a very good recovery and was discharged for rehabilitation 22 days after the operation. The patient did not manage very well with a prosthesis, but is very satisfied with crutches alone (Fig. 3).

\section{Discussion}

An aggressive surgical approach to sarcoma of the pelvic girdle offers the best chance of cure (Ravitch and Wilson, 1964). Hemipelvectomy as a form of treatment is used infrequently due to the extreme mutilation which results, although it may be the only way to help some patients. Douglass et al. (1975) describe the results of hemipelvectomy in 50 patients and stress that it is not a very complicated operation. This was confirmed by Miller (1977) who describes his experience with 126 cases. Mortality rates are also low. Miller reported a mortality rate of $1.5 \%$;

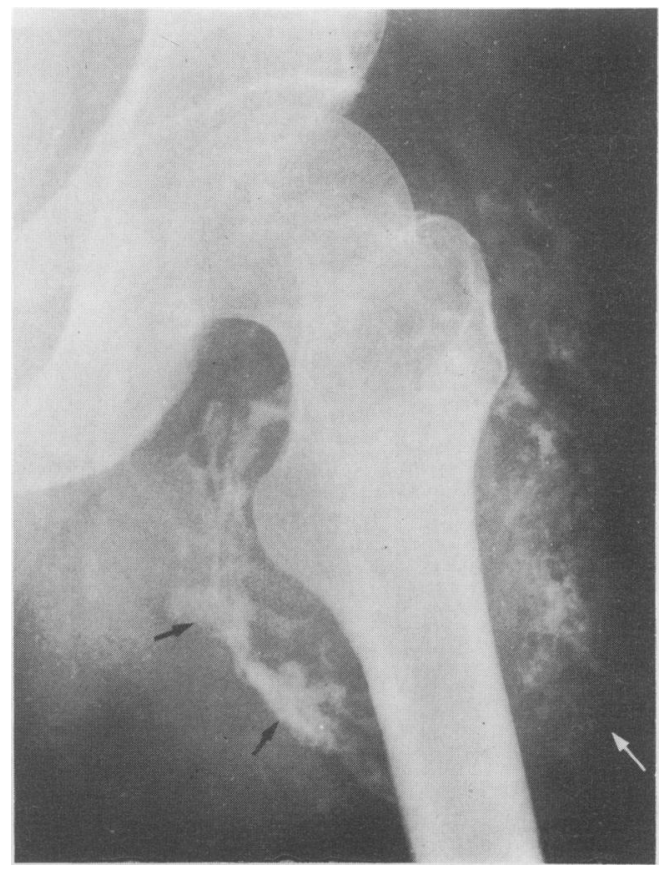

FIG. 2. Radiograph of the tumour of the left thigh showing the irregular areas of calcification.

Douglass et al. (1975) and Sneppen et al. (1978) in 41 cases had no mortality.

Murdoch (1969) and Sneppen et al. (1978) found that the rate of performance of the operation to be one per million population per year. This suggests that hemipelvectomy could be carried out more frequently in most areas.

The postoperative survival is relatively good. Miller (1977) reported an overall 33.3\% survival over 30 years, and Douglass et al. (1975) found 20\% of patients alive at 10 years. Sneppen et al. (1978) reported that there were 16 survivors, out of the 41 patients, who lived an average of 6.5 years. The remainder lived from 2 months to 7 years. Wu et al. (1977) found a 5 -year survival rate of $42.1 \%$ in a group of 19 patients.

Liposarcoma is a malignant tumour which is considered insensitive to radiotherapy and chemotherapy (Enterline et al., 1960). Wilbur (1975) suggested that some sarcomas respond with better results, if chemotherapy and radiotherapy are added following surgery. Stout (1944) has stated that some liposarcomas are definitely radiosensitive and that radiotherapy as a mode of treatment should not be neglected. Sensitization of the tumour by 5 -fluorouracil before radiotherapy induced a dramatic reduction in size in our case. The computerized tomography before treatment suggested that the tumour extended beyond the capabilities of surgery. From 


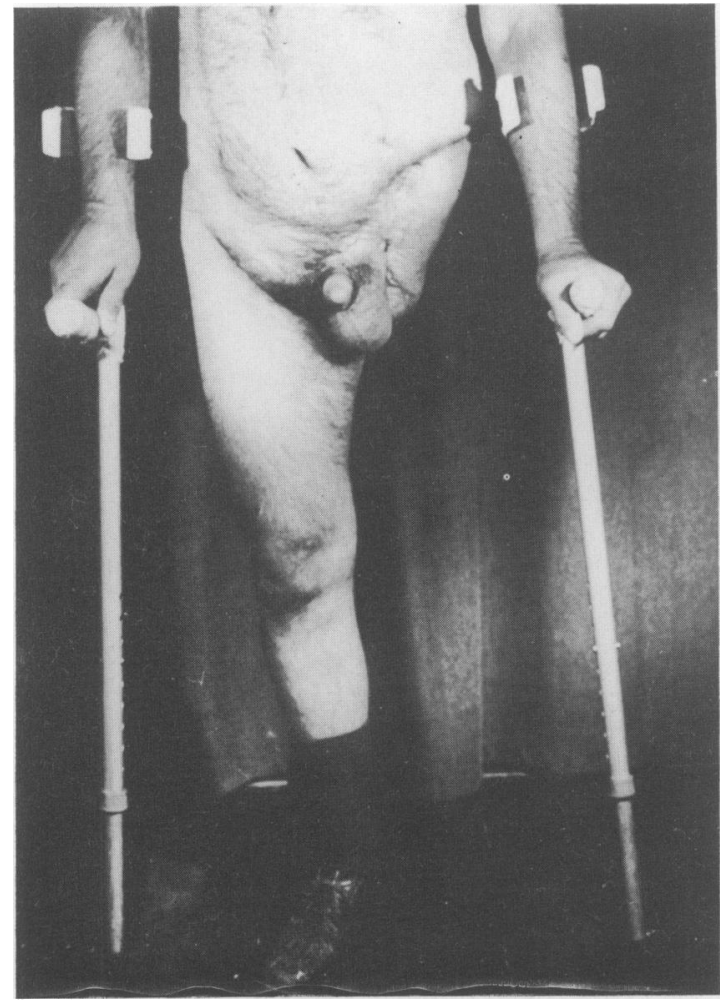

FIG. 3. Final result following left hemipelvectomy for liposarcoma. The patient preferred crutches to his prosthesis.

time to time cases which appear inoperable are allowed to deteriorate without radical therapy. In our own case there is a possibility that the initial assessment was incorrect and radical surgery should have been offered on presentation. However, it is more likely that the radiotherapy and chemotherapy converted a tumour which was inoperable, to one which was not only resectable, but possibly curable.

Rehabilitation of our patient proceeded satisfac- torily, although he preferred crutches to a prosthesi Reports of the use of a prosthesis after hemipelvec tomy vary considerably. Higinbotham and Cole 8 (1956) found that only two out of 25 cases used their prosthesis, whereas Miller (1959) reported 22 users: out of 32 cases. Sneppen et al. (1979) indicated that 15 out of 27 cases used the prosthesis but did poin out that eight patients out of 41 had mental problem? with one even committing suicide.

The results of surgery should be considered i⿱ terms of immediate goals of the surgical procedure control of the disease and functions. Hemipelvectomy is justifiable not only for complete cure but under some circumstances for palliation. Radiothe $\bar{\epsilon}$ apy and chemotherapy may make an inoperable tumour resectable or even curable. Patients cam function satisfactorily after the operation eve? though the results appear rather mutilating.

\section{References}

Douglass, H.O., RaZACK, M. \& HolyoKe, E.D. (1975) Hemipe? vectomy. Archives of Surgery, 110, 82.

ENTERLINE, H.T., Culberson, J.D., Rechlin, D.B. \& BRANDY, L.W. (1960) Liposarcoma: A clinical and pathological study of $\$ 3$ cases. Cancer, 13, 932.

Higinbotham, N.L. \& Coley, B.L. (1956) Hemipelvectomy: Expe ience in a series of thirty-nine cases. Cancer, 9, 1233.

KREMENTZ, E.T. \& SHAVER, J.O. (1963) Behavior and treatment of soft tissue sarcomas. Annals of Surgery, 157, 770.

MilleR, T.R. (1977) Hemipelvectomy in lower extremity tufor Surgical Clinics of North America, 8, 903. MURDOCH, G. (1969) Prosthetic and Orthotic Practice, p. 1 1े
Edward Arnold Ltd., London.

RaViTCH, M.M. \& WiLSON, T.C. (1964) Long term results of hemipelvectomy. Annals of Surgery, 159, 667.

Sneppen, O., Johansen, T., Heerfordt, J., Dissing, I., \& Petep足 SEN, I. (1978) Hemipelvectomy. Acta orthopaedica scandinavicळ 49, 175.

STOUT, A.P. (1944) Liposarcoma the malignant tumor of lipoplast Annals of Surgery, 119, 86.

WILBUR, J.R. (1975) Chemotherapy of sarcomas. Cancer, 36, 765. WU, K.K., Guise, E.R., Frost, H.M. \& Mitchell, L. (1977) The. surgical technique for hind-quarter amputation. Acta orthopaedicy scandinavica, 48, 479.

(Accepted 6 April 1983) 